\title{
Ultrafast micromachining of $\mathrm{Cu}$ and $\mathrm{Si}$ at ultra-high repetition rates with pulse bursts
}

\author{
Can Kerse ${ }^{1}$, Hamit Kalaycıoğlu², Parviz Elahi ${ }^{2}$, Koray Yavuz ${ }^{2}$, Inam Mirza ${ }^{3}$, Nadezhda M. \\ Bulgakova $^{3,4}$, F. Ömer Ilday ${ }^{1,2,5}$ \\ 1. Department of Electrical and Electronics Engineering, Bilkent University, Ankara, 06800, Turkey \\ 2. Department of Physics, Bilkent University, Ankara, 06800, Turkey \\ 3. HiLASE Centre, Institute of Physics ASCR, v.v.i, Dolní Břežany, Czech Republic. \\ 4. Institute of Thermophysics SB RAS, 630090, Novosibirsk, Russia \\ 5. UNAM - Institute of Materials Science and Nanotechnology, Bilkent University, Ankara, 06800, Turkey
}

\begin{abstract}
We report a novel ultrafast burst mode fiber laser system, which can deliver pulses at ultra-high repetition rates in order to systematically investigate micromachining efficiency on copper and silicon samples.
\end{abstract}

\section{INTRODUCTION}

The use of pulsed lasers has stimulated a growing interest in micromachining of different materials. Pulsed laser ablation is a well-known technique for micro-processing of materials. The mechanism of laser-material interaction for picosecond and femtosecond regime is quite different as compared to continuous wave and even nanosecond lasers. In ultrashort pulse interaction with material the pulse width is less then electron-lattice thermalization time (which is in the order of few ps for most of the metals). This feature drives the material into an extremely excited state followed by rapid quenching which leads to a material blow of composed of fast ions, atoms, clusters and nano-fragments of the ablated material before sufficient thermal diffusion takes place. This ablation regime brings many advantages for material processing such as reduction of heat affected zone and minimum collateral damage. Efficiency of material processing in ultrafast regime increases dramatically with repetition rate of the pulses, if the conditions are adjusted correctly to avoid excessive plasma and particulate shielding [1]. However, given that there is a minimum pulse energy requirement, continuous operation at high repetition rates can be detrimental due to too much average power leading to heat accumulation. Burstmode operation of lasers, wherein the amplifier periodically produces a group of pulses avoids this problem. Ultrafast burst-mode lasers are typically limited to several $100 \mathrm{MHz}$ intra-burst repetition rates. Although this is sufficient for most of the materials, metals with high thermal conductivity might require higher repetition rates.

Here, we demonstrate an all-fiber Yb burst mode laser amplifier system in order to systematically investigate the ablation efficiency at very high repetition rates, previously unexplored regime, with an in-burst repetition rate of 1728, 864, 432, 216 and 108 $\mathrm{MHz}$; which is optimized for low burst repetition rate.

\section{MATERIALS AND METHODS}

The system is able to produce down to 20-ns long bursts with a total energy of $175-210 \mu \mathrm{J}$ at a burst repetition rate of $1 \mathrm{kHz}$, and the individual pulses are compressed down to the sub-picosecond level. The seed signal from a $108 \mathrm{MHz}$ fiber oscillator is converted up to $1728 \mathrm{MHz}$ as multiples of $108 \mathrm{MHz}$ by a multiplier consisting of at five cascaded 50/50 couplers, and then amplified in nine stages (Fig. 1).

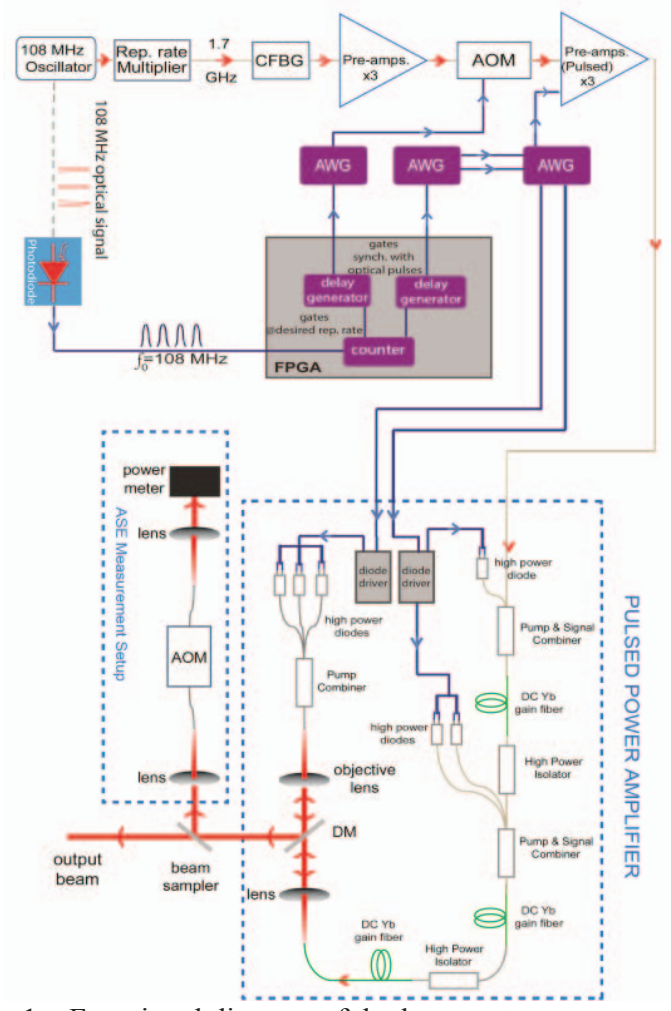

Fig. 1 - Functional diagram of the laser system

The highly cascaded amplification aims to suppress ASE at low repetition rate. Pulsed pumping synchronized with the seed by an FPGA-based electronic system is employed in the six latter amplifier stages. Pump pulses are optimized to obtain the highest 

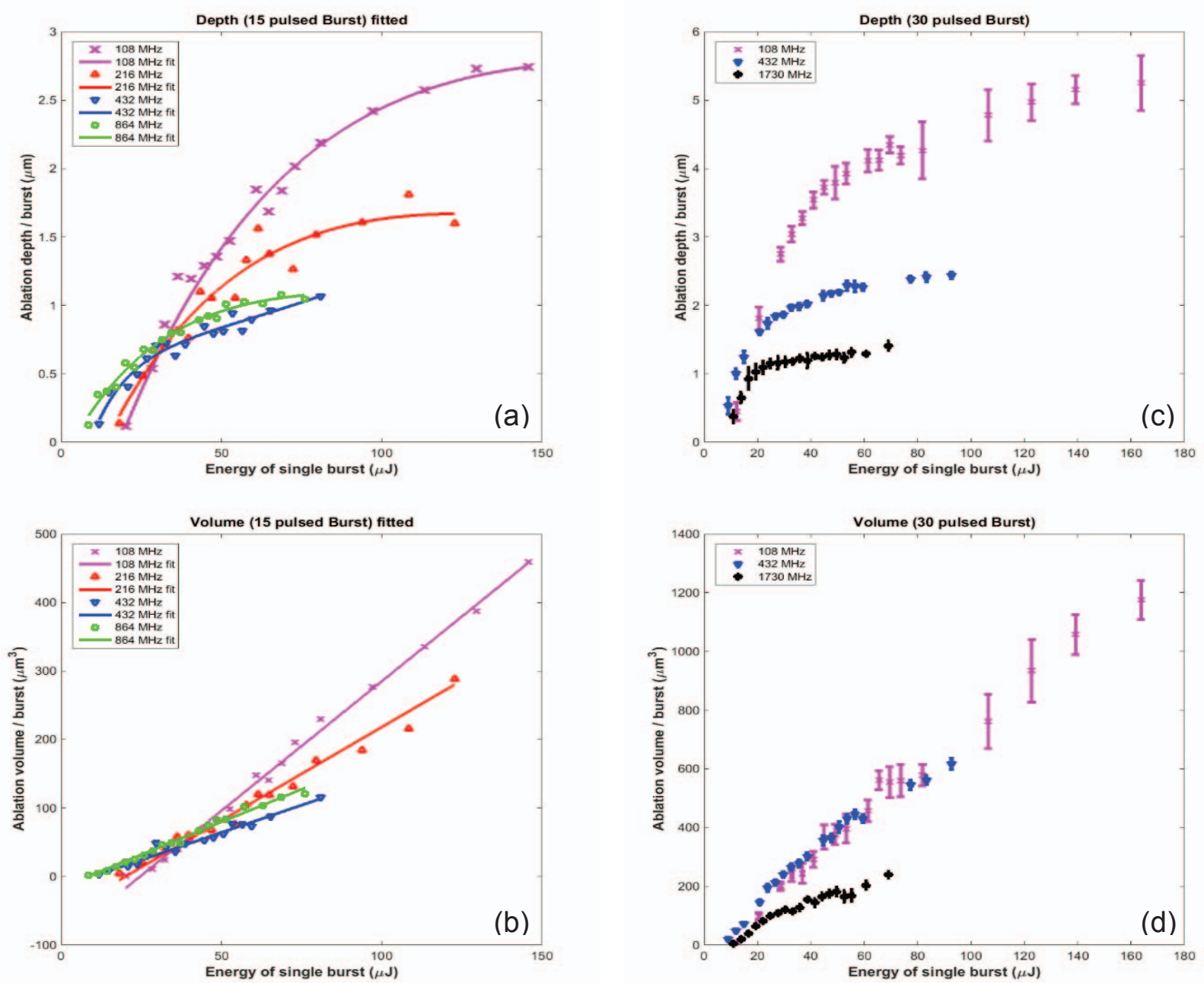

Fig. 2 - Ablation volume and depth vs. burst energy for several in-burst repetition rates. (a) Ablation depth/burst for copper. (b) Ablation volume/burst for copper. (c) Ablation depth/burst for silicon. (d) Ablation volume/burst for silicon

signal-to-ASE ratio, while ASE is monitored closely at the output of the system. At $1 \mathrm{kHz}$ burst repetition rate the ASE ratio for 30 pulsed burst operation is between $25 \%$ and $10 \%$ for $1728 \mathrm{MHz}$ and $108 \mathrm{MHz}$ respectively. The amplified bursts contain about 15 35 pulses of $5 \mu \mathrm{J}$ average and $8 \mu \mathrm{J}$ maximum individual energy. Previously, we have observed significant increases of efficiency with in-burst rates of 100 and $500 \mathrm{MHz}$ compared to the uniform repetition rate [2].

\section{RESULTS}

Fig. 2 shows ablation depth and volume per burst energy (bursts constituting $\sim 15$ pulses for copper and $\sim 30$ pulses for silicon) for different pulse repetition rates inside a burst. Ablation volume and depth seem to increase below $35 \mu \mathrm{J} /$ burst $(\sim 2 \mu \mathrm{J} /$ pulse $)$ as repetition rate increases, for copper samples. However, as repetition rate increases, the volume and depth of ablation decreases for silicon samples.

\section{CONCLUSION}

In conclusion, we built a custom burst mode fiber laser system, which is optimized for material processing at ultra-high repetition rates. In addition, we present, to our knowledge, the first investigation of this unexplored regime. We will continue our investigations on the mechanism of ablation in this regime.

\section{ACKNOWLEDGMENT}

Can Kerse acknowledges full Ph.D. scholarship from TÜBITAK BIDEB 2211.

\section{REFERENCES}

[1] W. Hu, Y. C. Shin, and G. King, "Modeling of multi-burst mode pico-second laser ablation for improved material removal rate," Applied Physics A, vol. 98, no. 2, pp. 407-415, 2010.

[2] C. Kerse, H. Kalaycıoğlu, O. F. Ilday, and E. Atalar, "Nonthermal Material and Tissue Processing with $100 \mathrm{MHz}$ and 500 MHz Repetition Rate Bursts," CLEO Europe-OSA Technical Digest (CD)(Optical Society of America, 2013), paper CM-P. $S$, vol. 26, 2013. 